\title{
Evaluation of natural language processing from emergency department computerized medical records for intra-hospital syndromic surveillance
}

Solweig Gerbier ${ }^{1,2^{*}}$, Olga Yarovaya ${ }^{2}$, Quentin Gicquel ${ }^{2}$, Anne-Laure Millet ${ }^{3}$, Véronique Smaldore ${ }^{3}$, Véronique Pagliaroli ${ }^{4}$, Stefan Darmoni ${ }^{5}$ and Marie-Hélène Metzger ${ }^{1,2}$

\begin{abstract}
Background: The identification of patients who pose an epidemic hazard when they are admitted to a health facility plays a role in preventing the risk of hospital acquired infection. An automated clinical decision support system to detect suspected cases, based on the principle of syndromic surveillance, is being developed at the University of Lyon's Hôpital de la Croix-Rousse. This tool will analyse structured data and narrative reports from computerized emergency department (ED) medical records. The first step consists of developing an application (Urglndex) which automatically extracts and encodes information found in narrative reports. The purpose of the present article is to describe and evaluate this natural language processing system.
\end{abstract}

Methods: Narrative reports have to be pre-processed before utilizing the French-language medical multiterminology indexer (ECMT) for standardized encoding. Urglndex identifies and excludes syntagmas containing a negation and replaces non-standard terms (abbreviations, acronyms, spelling errors...). Then, the phrases are sent to the ECMT through an Internet connection. The indexer's reply, based on Extensible Markup Language, returns codes and literals corresponding to the concepts found in phrases. Urglndex filters codes corresponding to suspected infections. Recall is defined as the number of relevant processed medical concepts divided by the number of concepts evaluated (coded manually by the medical epidemiologist). Precision is defined as the number of relevant processed concepts divided by the number of concepts proposed by Urglndex. Recall and precision were assessed for respiratory and cutaneous syndromes.

Results: Evaluation of 1,674 processed medical concepts contained in 100 ED medical records (50 for respiratory syndromes and 50 for cutaneous syndromes) showed an overall recall of 85.8\% (95\% Cl: 84.1-87.3). Recall varied from $84.5 \%$ for respiratory syndromes to $87.0 \%$ for cutaneous syndromes. The most frequent cause of lack of processing was non-recognition of the term by UrgIndex (9.7\%). Overall precision was $79.1 \%$ (95\% Cl: $77.3-80.8)$. It varied from $81.4 \%$ for respiratory syndromes to $77.0 \%$ for cutaneous syndromes.

Conclusions: This study demonstrates the feasibility of and interest in developing an automated method for extracting and encoding medical concepts from ED narrative reports, the first step required for the detection of potentially infectious patients at epidemic risk.

\footnotetext{
* Correspondence: solweig.gerbier-colomban@chu-lyon.fr

'Hospices Civils de Lyon, Hôpital de la Croix-Rousse, Unité d'hygiène et d'épidémiologie, F-69317 Lyon, France

Full list of author information is available at the end of the article
} 


\section{Background}

The prevention of nosocomial infections [1] must take into account the nosocomial risk of managing patients admitted to hospital with a community-acquired infection that poses an epidemic hazard. Identifying these patients upon admission would allow early implementation of precautionary measures in the admitting departments. Most frequently, patients admitted to hospital with a community-acquired infection first go to the emergency departments (ED). At this stage, they present with one or more symptoms expressed as a chief complaint. The diagnoses made at the end of these patients' clinical, biological and therapeutic management in EDs are often based solely on the physicians' best judgement, and are rarely confirmed by microbiological tests, which provide definitive results 24-48 hours after their receipt in the laboratory. This is why the early identification of patients admitted for a communityacquired infection that poses an epidemic risk should be based on syndromic surveillance.

Syndromic surveillance "focuses on the early symptom (prodrome) period before clinical or laboratory confirmation of a particular disease and uses both clinical and alternative data sources. Strictly defined, syndromic surveillance gathers information about patients' symptoms (e.g., cough, fever, or shortness of breath) during the early phases of illness" [2].

Few studies have investigated the surveillance of patients admitted to hospital with a community-acquired infection that poses an intra-hospital epidemic risk [3]. Most syndromic surveillance systems based on ED data are designed to identify anomalous phenomena (e.g., bioterrorism, emerging infectious disease) occurring within the community at a regional or even national level [4-13], but these methods have not been applied in intra-hospital settings to identify patients who represent an epidemic risk. Most of the systems described in the literature are based on the chief complaint [4-7,14] and sometimes on the syndromic discharge diagnosis [12,13]. In France, EDs are gradually computerizing their clinical records to meet the legislative framework for cooperation with the French National Institute for Public Health Surveillance (Institut de Veille Sanitaire, InVS) and regional health agencies for the transmission of health information [15]. The French Society of Emergency Medicine (Société Française de Médecine d'Urgence, SFMU) recommends encoding ED discharge diagnoses with the International Statistical Classification of Diseases and Related Health Problems, $10^{\text {th }}$ Revision (ICD-10), and chief complaints based on a thesaurus developed by the SFMU from a relevant selection of ICD-10 codes [16,17].

An automatic clinical decision support system for detecting patients carrying infections with an epidemic risk who are admitted to EDs is being developed at
Hôpital de la Croix-Rousse in Lyon. This detection tool will rely on computerized ED medical records (Dossier Médical des Urgences, DMU). These records contain early clinical data before any diagnostic confirmation is entered in real time (chief complaint, clinical examination data, etc.). The data entered in the DMU are heterogeneous and appear partly as structured variables and partly as textual variables corresponding to sections of narrative reports in medical language. An important part of the information needed for the syndromic identification of patients is described in the narrative reports that are divided into different sections: doctors' clinical observations, specialists' notes, prescribed diagnostic and therapeutic procedures. Each narrative report section is defined as a textual variable in the DMU database. Processing these narrative reports is a prerequisite for using DMU data.

The purpose of this paper was to describe and evaluate a natural language processing system to extract and encode information found in the narrative reports of computerized ED medical records.

\section{Methods}

\section{Source of DMU data}

The DMU is an integral part of the information system of the Lyon University Hospital. It contains numerous clinical data, some as structured variables (age, sex, type of admission, vital signs upon arrival, discharge mode, etc.), and others as textual variables (chief complaint, observation, diagnoses, etc.). This information is entered in real or near-real time. The DMU is also linked to the hospital's administrative database (age, sex, postal code, etc.).

A data warehouse extracts data from different modules of the hospital information system (DMU, administrative information system) and re-compiles them in the form of computerized reports. The content of these computerized reports (i.e., the choice of which variables to be extracted) is pre-defined by users. Computerized reports are generated with Business Object software in the form of Excel worksheets.

\section{Infectious syndromes targeted for identifying patients who pose an epidemic risk}

The infectious syndromes studied were:

- Cutaneous syndromes: skin infections that represent an epidemic risk (e.g., varicella, scabies, erysipelas);

- Gastrointestinal syndromes: infectious gastroenteritis (mainly viral), Clostridium difficile diarrhoea;

- Flu-like syndromes: viral respiratory infections (e.g., myxovirus (influenza), respiratory syncytial virus, parainfluenza virus);

- Meningeal syndromes: viral or bacterial meningitis; 
- Respiratory syndromes: upper (e.g., streptococcal angina) and lower (e.g., bronchitis, pneumonia, tuberculosis, whooping cough) respiratory tract infections.

Data selection for processing was based on a list of pre-established clinical concepts corresponding to the various infectious syndromes studied.

\section{French-language medical multi-terminology indexer}

To process the DMU's medical language, it was necessary to employ standardized medical terminology [18]. A French-language medical multi-terminology indexer (ECMT) developed by the CISMeF (Catalogue and index of French-language medical sites) is already available to the scientific community [19]. This indexer is based on algorithms that process medical terms from various terminologies by applying standardized codes of these terminologies. There are 24 terminologies currently integrated into the indexer, including the ICD-10, the French Common Classification of Medical Procedures (Classification Commune des Actes Médicaux, CCAM), the Systematized Nomenclature of Medicine, version 3.5 (SNOMED 3.5), the Anatomical, Therapeutic and Chemical (ATC) classification system, Medical Subject Headings (MeSH), the International Classification of Primary Care (ICPC-2) and the Dictionary of Consultation Results (DCR). Algorithms for this indexer are derived from those employed by the Doc'CISMeF search engine, developed by the same team [20].

The ECMT contains 2 types of query response modules: the default response module (descriptor), based on a bagof-words algorithm, and an extended response module (expansion), based on textual indexing, with Oracle Text ${ }^{\circledR}$ [21]. When querying a medical term, the "descriptor" response will return all concepts and codes corresponding to the different terminologies with at least 1 label matching all the query terms. The "expansion" module returns all concepts and corresponding codes with at least 1 label that contains the query terms and whose calculated score is greater than a threshold determined by the number of words found in the query. The number of words returned by the extended reply module is greater than the number returned by the default reply module. The indexer's URL is: http://ecmt.chu-rouen.fr/.

The query can be made directly on the Internet with the above URL or indirectly with an interface application that accesses the URL through an Internet connection. For the purpose of inter-operability, the indexer's reply is based on the XML (Extensible Markup Language) format. In this case, the ECMT module is callable from any medical questionnaire via a XML service.

\section{UrgIndex development method}

For syndromic identification, textual variables must first be processed in coded form based on medical terminologies.
An application called UrgIndex, which automatically processes DMU natural language data needed for syndromic surveillance, has been developed under Access with Visual Basic. More specifically, this application transforms medical concepts written in natural language into standardized codes after pre-processing of textual variables found in the DMU. A servlet developed for another project [21] submits textual variables to the ECMT to obtain the corresponding terminology codes. UrgIndex then filters the codes of suspected infection concepts, specifically identifying patients who pose an epidemic risk. Finally, the application records, displays and prints processed data for each patient.

\section{UrgIndex evaluation method}

Evaluation focuses on the quality of extracting and encoding the medical concepts found in DMU textual variables which are necessary for detecting at-risk patients.

\subsection{Adding a manual function to UrgIndex for building gold-standard extraction and encoding}

An additional function has been added to UrgIndex, making it possible to manually process natural language data. This function allowed the validation of data automatically processed, to code concepts that have been missed by the automatic process and to delete codes that have been incorrectly attributed by the automated process of the application. Validation was performed by a medical epidemiologist. Manually-validated medical concepts were considered as the gold standard for evaluating the automated process of textual variable extraction and encoding.

\subsection{Study population for evaluation}

Evaluation at the end of the learning phase The study population was selected from among adult patients who were admitted to the ED of 1 complex of the University of Lyon's Hôpital de la Croix-Rousse. This facility has 810 beds. As UrgIndex development was the first step necessary for building detection algorithms of at-risk patients, we needed to evaluate the performance of automated natural language processing. To this end, we randomly selected 50 patients with hospital-confirmed diagnoses of infection corresponding to 1 of the 5 syndromic syndromes of interest. This random selection was made from a retrospective cohort of 8,958 patients hospitalized, at the conclusion of their emergency visit in Hôpital de la Croix-Rousse between January 1, 2008, and March 31, 2010. The cohort was selected for development of the complete automatic clinical decision support system. The number of 50 infected patients was reached for cutaneous infections and respiratory tract infections. The number of selected patients was lower than 50 in this cohort for gastrointestinal infections $(\mathrm{n}=18)$, flu-like syndromes $(\mathrm{n}=$ $21)$ and meningeal infections $(\mathrm{n}=19)$. Totally, 158 medical records served to develop the application and to 
evaluate processing quality obtained at the end of the learning phase.

Evaluation in the test phase As UrgIndex filters were completed with the 158 medical records described above, a new, random selection of infected patients was made in the retrospective cohort for test phase evaluation. Due to an insufficient number of infected patients in our cohort, evaluation was performed only on 2 syndromes: 50 medical records of patients with respiratory syndromes and 50 medical records of patients with cutaneous syndromes. Totally, 100 medical records were considered for evaluation.

\subsection{Evaluation indicators}

Recall was defined as the number of relevant, processed medical concepts (true positives) proposed by UrgIndex divided by the expected number of medical concepts evaluated (coded manually by the medical epidemiologist). Precision was defined as the number of relevant processed concepts (true positives) divided by the total number of medical concepts proposed by UrgIndex.

Recall and precision were calculated globally and separately for each type of textual variable (sections of the narrative report): "reason," "clinical observations," "specialists' notes," "biological procedures and diagnoses other than biological and therapeutic" and "discharge prescriptions." 95\% confidence intervals ( $95 \% \mathrm{CI})$ for recall and precision (Clopper-Pearson interval for binomial proportion) were computed by $\mathrm{R}$ software.

Non-coded concepts (false negatives) were classified according to the following categories: 1) ECMT inadequacy (missing code or phrase too long to manage); 2) language variations not supported by the ECMT; 3) missing code for application filters; 4) negation mismanaged by the application; and 5) other application anomalies.

\section{Results}

\section{Presentation of UrgIndex and the natural language data processing circuit}

Figure 1 depicts automated textual processing by UrgIndex.

\subsection{Launching natural language data processing}

In this first experimental phase, data processing is launched manually. DMU natural language data are imported manually from the data warehouse in Excel format (table "report" in Figure 1) according to a pace decided by the user. In the first step (step 1, Figure 1), only textual variables corresponding to narrative sections of the medical reports are imported from the table "report". Patients were selected for this experimental phase (step 2, Figure 1). A new table "report_twice" was created in the application.

\subsection{Automated textual processing by UrgIndex}

Some textual DMU variables (corresponding to sections of the medical narrative report, such as clinical observations and specialists' notes) are long textual variables consisting of a large number of characters (sometimes more than 2,000). Free text sometimes consists of complex phrases or groups of words describing a patient's clinical condition, his/her history, the history of his/her current pathology and management in EDs.

The textual variable has to be pre-processed before it is sent to the ECMT (step 3, Figure 1). UrgIndex partitions the text into 2 steps. The first step is to split the textual variable into sentences. This sectioning is done by tracking periods followed by a space or when 2 groups of words are separated by a line break. The second step is to partition each sentence into syntagmas. Partitioning is done after looking for punctuation marks (question marks, exclamation marks, commas, parentheses, ellipses, and semicolons), coordinating conjunctions and prepositions (and, but, or, therefore, however, neither, nor, because, and with), which are previously listed and stored in an Access table. After partitioning the phrases into syntagmas, UrgIndex performs the following procedures: 1) It identifies negations in the syntagmas (different negation methods - no, not, nor, none, lack of, lack, lacking, of absence, absence of, the absence, devoid of, does not, did not, didn't, doesn't, is not, isnot, isn't, isnt, has not received, has not received any, has not, destitute of, devoid of, never - are listed and stored in an Access table). Syntagmas containing the identified negations are removed from the phrase; 2) It recognizes non-standard terms (abbreviations, acronyms, spelling errors and synonyms not recognized by the ECMT - all these terms having been listed and stored in an Access table showing correspondence between the terms and an ECMT terminology label) and replaces them with a corresponding ECMT term; 3) It spots and removes numerical values in the phrase (numerical values are otherwise recognized as terminology codes by ECMT which offers irrelevant labels); 4) It re-concatenates different syntagmas from the same phrase. Re-concatenations of different phrases extracted from the same textual variables are temporarily stored in a separate Access table called "phrase".

Figure 2 illustrates the automated processing of a textual variable (clinical observation) of a patient who had the flu. The narrative section was partitioned into syntagmas at punctuations ("comma" in this example), conjunctions ("and") or prepositions ("with"). Unrecognized abbreviations corresponding to medical concepts ("cgh" for "cough") or unrecognized synonyms ("aches" for "myalgia") were automatically replaced by UrgIndex with medical concepts recognized by the ECMT. A syntagma containing a negation ("no sore throat") was excluded. The value "39" was deleted. The syntagmas were re-concatenated, and the phrase "patient presents with flu syndrome, severe onset of symptoms, fever over $\mathrm{C}^{\circ}$, myalgia and dry cough, asthenia" was ready to be sent to the ECMT. 


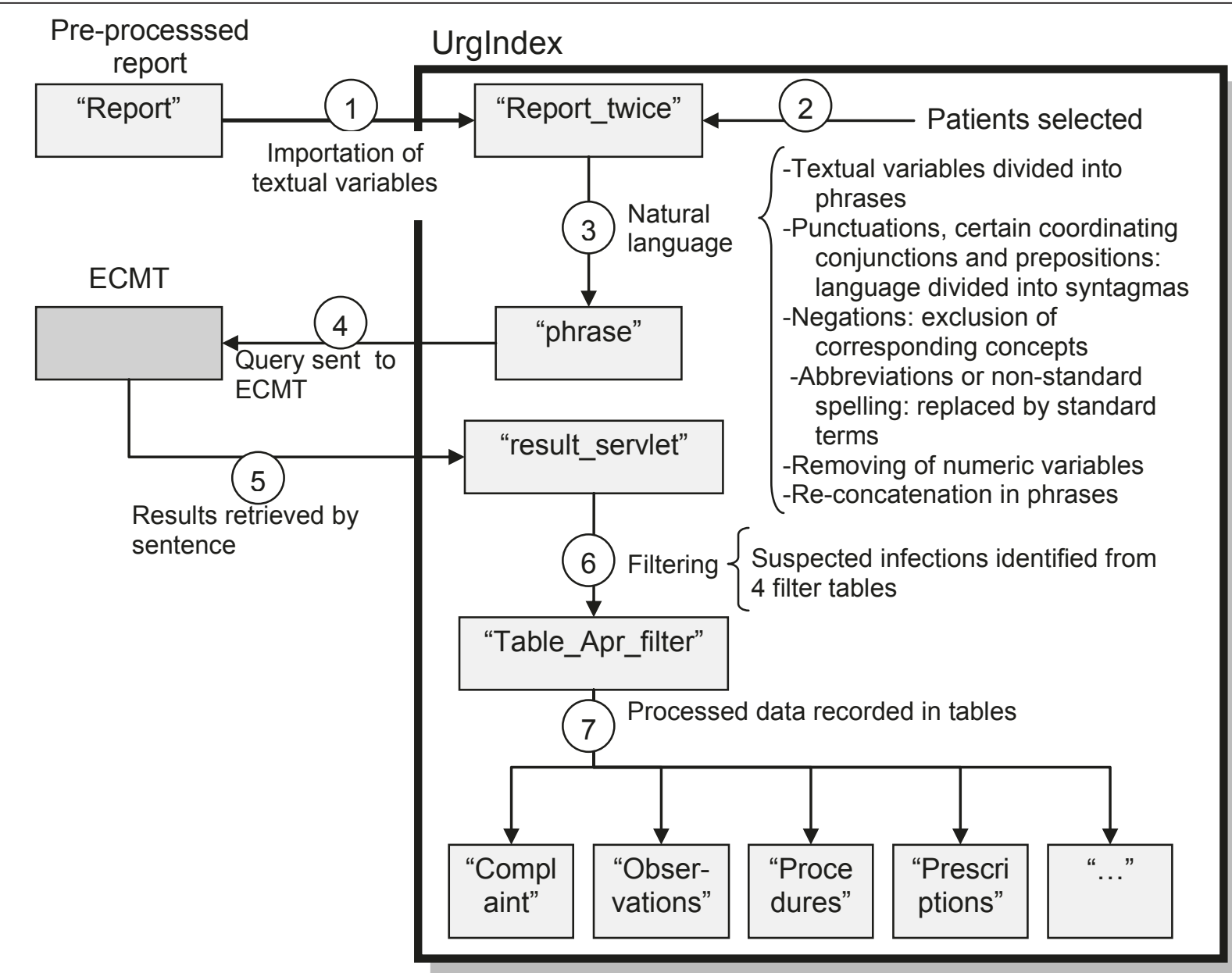

Figure 1 Processing of natural language data extracted from emergency department medical records by UrgIndex (University of Lyon's Hôpital de la Croix-Rousse, Lyon, France).

Another example with a longer narrative section of the observation is shown in Figure 3.

\subsection{Sending and retrieving terminology codes via the ECMT}

Once the textual variable is pre-processed, the re-concatenated phrases are conveyed to the ECMT via the servlet. Each re-concatenated phrase is dispatched automatically to the ECMT one after another to obtain the corresponding terminology codes (step 4, Figure 1). Each label obtained with the code and terminology is retrieved by UrgIndex and stored in a temporary table "result_servlet" (step 5, Figure 1).

ECMT terminologies were chosen according to the medical narrative section processed. The following terminologies were selected:

- "chief complaint," "observation," "specialists' notes," "discharge diagnosis labels": ICD-10, SNOMED 3.5, DCR, ICPC-2, MeSH, ATC and CCAM;

- "biological procedures" and "other diagnoses: CCAM, MeSH, SNOMED 3.5;
- "therapeutic procedures" and "discharge prescriptions": ATC, MeSH.

Only codes retrieved by the ECMT and corresponding to relevant terminologies for the narrative section were filtered by UrgIndex.

\subsection{Selecting medical concepts related to a suspected} infection

UrgIndex will be integrated in an automatic clinical decision support system aimed at identifying patients who pose an epidemic risk. Therefore, UrgIndex should retain, at the end of natural language processing, only medical concepts relating to one of the studied syndromes. A second filtering makes it possible to retain only suspected infection concepts. Four specific filters were created for each type of narrative section:

- Filtering of symptoms (filtering chief complaints, observations, specialists' notes, discharge diagnosis);

- Filtering of biological diagnostic procedures; 


\section{SOURCE: Exemple of observation with 1 phrase \\ Patient presents with flu syndrome, severe onset of symptoms, fever over $39 \mathrm{C}^{\circ}$, aches and dry $\underline{\operatorname{cgh}}$, asthenia, no sore throat

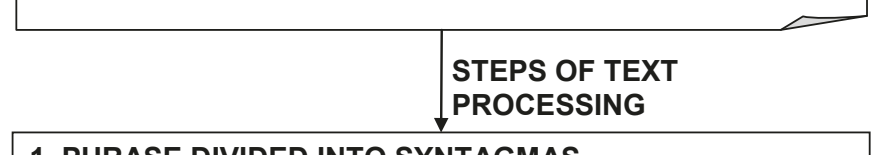

\section{PHRASE DIVIDED INTO SYNTAGMAS}

Patient presents with flu syndrome, severe onset of symptoms, fever over $39 \mathrm{C}^{\circ}$, aches and dry $\underline{c g h}$, asthenia, no sore throat
Terms not recognized by the ECMT are

- synonym not processed by the ECMT (Italics)

- Abbreviation (Underlined italics) Negation (Bold)

2. NEGATIONS SOUGHT ("no sore throat" syntagma deleted) 3. SPELLING AND ABBREVIATION REPLACED

4. NUMERIC VALUES REMOVED $\left(39^{\circ} \mathrm{C}\right)$

Patient presents

with flu syndrome,

severe onset of symptoms,

fever over $\mathrm{C}^{\circ}$,

myalgia

and dry cough,

asthenia,

\section{PHRASE RE-CONCATENATED}

Patient presents with flu syndrome, severe onset of symptoms, fever over $\mathrm{C}^{\circ}$, myalgia and dry cough, asthenia,

\begin{tabular}{|c|c|}
\hline & $\begin{array}{l}\text { 6. RE-CONCATENATED PHRASE SENT } \\
\text { TO THE ECMT }\end{array}$ \\
\hline $\begin{array}{l}\text { 7. OBSERVATION PROCESSE } \\
\text { LABELS AND FILTERING SUS } \\
\text { "Observations" table : } \\
\text { SNO / DE-30110 / flu-like syndr } \\
\text { 11355_CRI / + - fever; CIP / A0 } \\
\text { F-24100 / cough; SNO / F-2411 } \\
\text { / 12905_CRI / ++++ cough; DR } \\
\text { asthenia; DRC / 10326_CRI / + }\end{array}$ & $\begin{array}{l}\text { ED IN URGINDEX AFTER RETRIEVING ECMT STANDARDIZED CODES AND } \\
\text { SPECTED INFECTION CODES } \\
\text { ome; DRC / 10781_CRI / + - severe onset; SNO / F-03003 / fever; DRC / } \\
3 \text { / fever; MSH / D005334 / fever; ICD / M79.1 / myalgia; ICD / R05 / cough; SNO / } \\
6 \text { / dry cough; DRC / } 12619 \text { _CRI / + - dry; DRC / } 12909 \text { CRI / + - dry cough; DRC } \\
\text { C / } 758 \text { / cough; CIP / R05 / cough; MSH / D003371 / cough; SNO / F-01380 / } \\
\text { - asthenia; MSH / D001247 / asthenia }\end{array}$ \\
\hline
\end{tabular}

Figure 2 Processing of a medical narrative report section by UrgIndex. Example of processing the narrative section "clinical observation" of a patient going to the emergency department of Hôpital de la Croix-Rousse (a University of Lyon hospital) with a flu-like syndrome.

- Filtering of diagnostic procedures other than biological;

- Filtering of "therapeutic procedures" and "discharge prescriptions".

The 4 filters were first implemented with clinical knowledge, and diagnostic and therapeutic care related to the disease targeted for this project. Then, the 4 filters were gradually supplemented by the epidemiologist, with 158 medical records of infected patients in the learning phase (50 cutaneous infections, 18 gastrointestinal infections, 21 flu-like syndromes, 19 meningeal infections and 50 respiratory tract infections).

The codes and labels obtained by the ECMT were filtered with these tables. After filtering, the codes and labels were stored in a temporary table ("aft-filter" table, 


\section{SOURCE : Example of observation with 3 phrases
Hyperthermia 38.3. Abdomen supple in general, without guarding \\ SOURCE : Example of observation with 3 phrases
Hyperthermia 38.3. Abdomen supple in general, without guarding nor splenomegaly, no disorders of gastrointestinal transit, no maelena, no hemorrhage of rectum, no hematemesis, decrease of the $\underline{V M}$ in bases, productive cough, no pharyngitis and no $\underline{C M}$ eruption, no purpura, no petechia. $\underline{C R}$ : diffuse bronchial $\underline{S d}$. \\ STEPS OF TEXT PROCESSING}

Terms not recognized by the ECMT are:

- synonym not processed by the ECMT (Italics)

- Abbreviation (underlined italics)

Negation (Bold)

\section{OBSERVATION DIVIDED INTO PHRASES, THEN PHRASES DIVIDED INTO SYNTAGMAS}

Phrase 1:

Hyperthermia 38.3.

Phrase 2:

Abdomen supple in general,

without defense nor splenomegaly,

no disorders of gastrointestinal transit,

no maelena,

no hemorrhage of rectum,

no hematemesis,

decrease of the $\underline{V M}$ in bases,

productive cough,

no pharyngitis

and no $\underline{\mathrm{CM}}$ eruption

no purpura,

no petechia.

Phrase 3:

$\underline{C R}$ : diffuse bronchial $\underline{S d}$.
2. NEGATIONS SOUGHT (syntagmas deleted: "without defense nor splenomegaly", "no disorders of gastrointestinal transit", "no maelena", "no hemorrhage of rectum", "no hematemesis", "no pharyngitis", and no CM eruption", "no purpura", "no petechia")

\section{SPELLING AND ABBREVIATION REPLACED}

4. NUMERICAL VALUES REMOVED $\left(39^{\circ} \mathrm{C}\right)$

Phrase 1:

Hyperthermia.

Phrase 2:

Abdomen supple in general,

decrease of vesicular murmur in bases,

productive cough,

Phrase 3:

Chest radiography: diffuse bronchial syndrome.

\section{PHRASES RE-CONCATENATED}

Phrase 1: Hyperthermia

Phrase 2: Abdomen supple in general, decrease of vesicular murmur in bases, productive cough,

Phrase 3: $\underline{\text { Chest radiography: diffuse bronchial syndrome. }}$

\section{RE-CONCATENATED PHRASE SENT} SEPARATLY TO THE ECMT

\section{OBSERVATION PROCESSED IN URGINDEX AFTER RETRIEVING ECMT STANDARDIZED CODES AND LABELS AND FILTERING SUSPECTED INFECTION CODES \\ "Observations" table: \\ SNO / F-03003 / fever; SNO / F-0A440/ hyperthermia; MSH / D005334 / fever; SNO / F-23010 / vesicular mumur; SNO / G-A316 / decreased; CIM / R05 / cough; SNO / F-24100 / cough; SNO / F-241C0 / Productive cough; DRC/ 12905_CRI / ++++ cough; DRC /758 / cough; CIP / R05 / cough; MSH / D003371 / cough; SNO / T-28000 / Lung, nos; SNO / P5-20000 / Radiography of chest, nos; MSH / D013577 / syndrome; SNO / T-26000 / Bronchus, nos; SNO / G-A321 / Diffuse}

Figure 3 Processing of a medical narrative report section by UrgIndex. Example of processing narrative section "clinical observation", when there is more than one phrase, of a patient going to the emergency department of Hôpital de la Croix-Rousse (a University of Lyon hospital) with a bronchitis.

step 6, Figure 1) and then divided into different tables corresponding to narrative sections of the report (reason, observation, procedures, etc.). These tables formed the ready-to-use database for the detection of patients at epidemic risk.
In the examples enumerated in Figures 2 and 3, all medical terms contained in the phrase were processed by the ECMT. The codes and their labels selected after the different filtering processes were stored in the table "observation" of UrgIndex, making it possible to exploit 
them for the next step, i.e. the detection algorithm of patients at-risk.

\section{Evaluation of the natural processing system to extract and encode information found in the narrative reports \\ 2.1. Evaluation at the end of the learning phase}

The 158 patient records selected for the learning phase of UrgIndex made it possible to assess 3,023 suspected infection concepts. The number of concepts evaluated differed from one textual variable to another: there were $204(6.7 \%)$ for the variable "chief complaint"; 2,625 (86.8\%) for "clinical observation"; 33 (1.1\%) for "specialists' notes"; 145 (4.8\%) for "biological procedures, diagnoses other than biological and therapeutic"; and 16 $(0.5 \%)$ for "discharge prescriptions." Of the 3,023 concepts, 2,593 (85.8\%; 95\% CI: 84.5-87.0) were correctly processed. Recall varied from 93.1\% (95\% CI: 89.7-96.7) for "chief complaint" to $36.4 \%$ (95\% CI: 22.2-54.5) for "specialists' notes". Overall, of the 430 unprocessed concepts, the reasons were: missing corresponding code in the ECMT for 104 concepts (24.2\%); missing code in the UrgIndex filters for 38 concepts (8.8\%); terms not recognized by UrgIndex (abbreviations, synonyms, spelling errors, acronyms not recognized in the correspondence tables) for 213 concepts (49.5\%); poor negation management by UrgIndex for 10 concepts (2.3\%); and other types of UrgIndex errors for 65 concepts (15.1\%).

UrgIndex recall for extracting and encoding medical concepts by type of syndrome varied from $81.3 \%$ (95\% CI: 77.8-84.9) for meningeal syndromes to $90.0 \%$ (95\% CI: 86.7-93.1) for gastrointestinal syndromes.

\subsection{Evaluation in the test phase}

From the 100 patient records selected for the test phase (50 with respiratory syndrome and 50 with cutaneous syndrome), 1,952 medical concepts were expected to be processed correctly by UrgIndex. There were 128 (6.6\%) concepts for the variable "chief complaint"; 1,736 $(88.9 \%)$ for "clinical observation"; $15(0.8 \%)$ for "specialists' notes"; 60 (3.1\%) for "biological procedures, diagnoses other than biological and therapeutic"; and 13 (0.7\%) for "discharge prescriptions."

Table 1 shows recall and precision for both syndromes separately and together.

Of 1,952 concepts, 1,674 were correctly processed (recall 85.8\%, 95\% CI: 84.1-87.3), while 443 concepts were wrongly proposed by UrgIndex (precision 79.1\%, 95\% CI: 77.3-80.8). Table 2 gives the reasons for unprocessed concepts by type of variable. Overall, of the 278 unprocessed concepts, the reasons were: missing corresponding code in the ECMT for 6 concepts (2.2\%); missing code in the UrgIndex filters for 17 concepts (6.1\%); terms not recognized by UrgIndex (abbreviations, synonyms, spelling errors, acronyms not recognized in the correspondence tables) for 190 concepts (68.3\%); poor negation management by UrgIndex for 9 concepts (3.2\%), and other types of UrgIndex errors for 56 concepts $(20.3 \%)$.

There were 443 non-relevant processed concepts proposed by UrgIndex (false positives). Table 3 gives the reasons for non-relevant processed concepts by type of variables. The reasons for non-relevant processed concepts were: they were related to antecedents, including pathologies, usual treatment or allergies for 157 concepts (35.4\%; 95\% CI: 31.0-40.1); the same concept concerned infectious and non-infectious disease for 257 concepts (58.0\%; 95\% CI: 53.3-62.7); the clinical sign was absent but UrgIndex did not detect the negation for 19 concepts (4.3\%; 95\% CI: 2.6-6.6); the abbreviation stood for a concept other than the one proposed for 7 concepts (1.6\%; 95\% CI: 0.6-3.2); and other UrgIndex anomalies for 3 concepts (0.7\%; 95\% CI: 0.1-2.0).

\section{Discussion}

In the early stage of patient management, syndromic surveillance is instrumental in preventing and controlling nosocomial epidemic phenomena related to the admission of patients who could be an epidemic risk. Identification is an important means of helping infection control practitioners implement preventive measures to limit the risk of transmission of infections that pose an epidemic risk, including additional precautions (contact, droplets, air), for interaction with the clinical teams. It is, therefore, important to implement tools to identify patients who represent a risk in EDs before they are even admitted. Knirsch et al. tested an automated clinical decision support system for identifying additional, potential tuberculosis patients who clinicians failed to place in respiratory isolation [3]. This tool was based on the use of a natural language processing system to encode narrative chest radiograph reports, called MedLEE (Medical Language Extraction and Encoding System) and algorithms checking laboratory and pharmacy data for evaluating the immunocompromised status of patients. Based on a retrospective cohort study conducted for evaluation in 1992-1993, the combination of clinical and automated clinical decision support systems improved the isolation rate from $62.6 \%$ to $78.4 \%$, disclosing the relevance of automated methodologies for detecting patients at risk.

A similar experiment is underway to develop an automated clinical decision support system at Hôpital de la Croix-Rousse. Natural language processing is a necessary prerequisite for this process. UrgIndex was designed to automatically process natural language data.

Evaluation of UrgIndex, which was part of its development, indicated that processing quality was satisfactory. Recall was $85.8 \%$, ranging from $81.3 \%$ to $90.0 \%$, depending on the type of syndrome at the end of the learning 
Table 1 Recall and precision of Urglndex in the test phase ( $\mathrm{n}=100$ medical records)

\begin{tabular}{|c|c|c|c|c|c|c|c|}
\hline & \multirow{2}{*}{$\begin{array}{l}\text { Number of medical } \\
\text { concepts evaluated }\end{array}$} & \multirow{2}{*}{$\begin{array}{l}\text { Number of correctly } \\
\text { processed concepts } \\
\text { (true positives) }\end{array}$} & \multirow{2}{*}{$\begin{array}{l}\text { Number of medical concepts } \\
\text { proposed by UrgIndex }\end{array}$} & \multicolumn{2}{|r|}{ Recall * } & \multicolumn{2}{|c|}{ Precision $\S$} \\
\hline & & & & (\%) & $\begin{array}{c}95 \% \\
\text { confidence } \\
\text { interval }\end{array}$ & $(\%)$ & $\begin{array}{c}95 \% \\
\text { confidence } \\
\text { interval }\end{array}$ \\
\hline $\begin{array}{l}\text { Respiratory } \\
\text { syndrome }\end{array}$ & 966 & 816 & 1,002 & 84.5 & $82.0-86.7$ & 81.4 & 78.9-83.8 \\
\hline $\begin{array}{l}\text { Cutaneous } \\
\text { syndrome }\end{array}$ & 986 & 858 & 1,115 & 87.0 & 84.8-89.1 & 77.0 & $74.4-79.4$ \\
\hline Total & 1,952 & 1,674 & 2,117 & 85.8 & $84.1-87.3$ & 79,1 & $77.3-80.8$ \\
\hline
\end{tabular}

Urglndex- Processing of emergency services medical narrative records (DMU) of the University of Lyon's Hôpital de la Croix-Rousse, France

${ }^{*}$ Recall $=$ Number of relevant processed concepts (true positives)/number of medical concepts evaluated (manually coded by the medical epidemiologist) $\S$ Precision $=$ Number of relevant processed concepts (true positives)/number of medical concepts proposed by Urglndex.

phase. Evaluation of recall on a new set of 100 medical records confirmed its good performances in terms of recall $(85.8 \%$ overall) and precision $(79.1 \%$ overall).

The small number of available concepts for "specialists' notes" and "discharge prescriptions" shows that these variables are seldom used by clinicians. For the variables "reasons," "observations" and "procedures", the lack of processing was linked mostly to the presence of either an abbreviation, acronym, synonym or spelling error unrecognized by the ECMT and not present in the UrgIndex correspondence table. This language variation table is an important UrgIndex asset for processing natural language data that are sometimes approximate (employing common words instead of medical words, regional words, abbreviations or unconventional acronyms or spelling or typing errors). Language variations are responsible of false negatives (not perfect recall) and to a lesser extent of false positive (not perfect precision). We should emphasize the particular difficulty of obtaining an exhaustive correspondence table, given the very telegraphic style of emergency physicians' notes and typing errors in the emergency context to trace the patient's clinical description. UrgIndex was designed to enrich this correspondence table as it was being utilized.

Another limitation is related to the ECMT. Some clinical concepts and their synonyms have no codes in the ECMT, as illustrated by "bronchial congestion," and "air bronchogram." Also, the same acronyms are sometimes applied to 2 different concepts, which are easily understandable in the context by a clinician, but may not be correctly interpreted by the ECMT. For example, the acronym "ARF" can mean both "acute respiratory failure" and "acute renal failure."

Finally, the application does not contextualize concepts found in textual variables based on their occurrence timeline and does not perform sustained semantic analysis. It is only based on a search of medical concept. This participates to the not perfect precision (79.1\%) as false positives due to antecedents represented $35 \%$ of all false positives in the test set. For example, the application does not distinguish if a symptom is an antecedent, belongs to the current history of the disease or corresponds to a current clinical examination. Such a limitation can lead to background noise (codes of suspected infection concepts for patients who do not have any; for example, "the patient had pulmonary tuberculosis in 1982": processing in the application will return the "pulmonary tuberculosis" code).

Background noise may be the source of false positives, which will require the validation of cases, within patients detected by the automated clinical decision support system, by infection control practitioners before alerting health care providers. A study is also being planned to determine the sensitivity/specificity of case identification by the clinical decision support system prior to its implementation in hospital. This evaluation will be carried out once the tool is fully developed (i.e. once the detection algorithms are completely developed with the DMU's structured data and textual data and fully integrated into the clinical decision support system).

Many authors have already expressed interest in syndromic surveillance in hospital EDs. Such surveillance is possible if medical records are computerized and permit regular computerized transmission of necessary data to epidemiological services in charge of this surveillance $[4-7,14]$. Most syndromic surveillance systems described in the literature are based on the surveillance of chief complaints $[4-7,14]$ or discharge diagnoses $[12,13]$ in EDs to detect potential outbreaks of target diseases as soon as possible, to provide early warning to the community if necessary and to incite epidemiological field investigations to confirm the diseases as well as their origin, and take appropriate measures. For example, a syndromic surveillance system was implemented in Virginia in 7 EDs for 10 months [7]. The chief complaints were faxed daily to the health department, classified manually according to 7 syndromes (fever, respiratory distress, vomiting, diarrhoea, rash, disorientation and sepsis), and analyzed by the 
Table 2 Evaluation of the processing quality of concepts by type of variable on the test set

\begin{tabular}{|c|c|c|c|c|c|c|c|c|c|c|c|c|c|c|c|c|c|c|}
\hline & \multicolumn{3}{|c|}{ Chief complaint } & \multicolumn{3}{|c|}{ Observation } & \multicolumn{3}{|c|}{ Specialists' notes } & \multicolumn{3}{|c|}{ Procedures } & \multicolumn{3}{|c|}{ Prescriptions } & \multicolumn{3}{|c|}{ Total } \\
\hline & $\mathrm{N}_{*}$ & $\%$ & $95 \% \mathrm{Cl}$ & $\mathrm{N}$ & $\%$ & $95 \% \mathrm{Cl}$ & $\mathrm{N}$ & $\%$ & $95 \% \mathrm{Cl}$ & $\mathrm{N}$ & $\%$ & $95 \% \mathrm{Cl}$ & $\mathrm{N}$ & $\%$ & $95 \% \mathrm{Cl}$ & $\mathrm{N}$ & $\%$ & $95 \% \mathrm{Cl}$ \\
\hline Correctly-processed concepts & 117 & 91.4 & $85.1-95.6$ & 1,481 & 85.3 & $83.6-86.9$ & 11 & 73.3 & $44.9-92.2$ & 53 & 88.3 & $77.4-95.2$ & 12 & 92.3 & $64.0-99.8$ & 1,674 & 85.8 & $84.1-87.3$ \\
\hline Missing code in the ECMT $\S$ & 0 & - & - & 6 & 0.3 & $0.1-0.8$ & 0 & - & - & 0 & - & - & 0 & - & & 6 & 0.3 & $0.1-0.7$ \\
\hline Missing code in UrgIndex filters & 0 & - & - & 16 & 0.9 & $0.5-1.5$ & 0 & - & - & 0 & - & - & 1 & 7.7 & $0.2-36.0$ & 17 & 0.9 & $0.5-1.4$ \\
\hline Term not recognized by UrgIndex $†$ & 6 & 4.7 & $1.7-9.9$ & 176 & 10.1 & 8.8-11.7 & 1 & 6.7 & $0.2-31.9$ & 7 & 11.7 & 4.8-22.6 & 0 & - & & 190 & 9.7 & $8.5-11.1$ \\
\hline Negation not recognized by UrgIndex & 0 & - & - & 9 & 0.5 & $0.2-1.0$ & 0 & - & - & 0 & - & - & 0 & - & & 9 & 0.5 & $0.2-0.9$ \\
\hline Other UrgIndex error & 5 & 3.9 & $1.3-8.9$ & 48 & 2.8 & $2.0-3.6$ & 3 & 20 & $0.4-48.1$ & 0 & - & - & 0 & - & & 56 & 2.9 & $2.2-3.7$ \\
\hline Total number of concepts & 128 & 100 & - & 1,736 & 100 & - & 15 & 100 & - & 60 & 100 & - & 13 & 100 & - & 1,952 & 100 & - \\
\hline
\end{tabular}

UrgIndex- Processing of emergency services medical narrative records (DMU) of the University of Lyon's Hôpital de la Croix-Rousse, Lyon, France

*N $=$ Number of evaluated concepts

SECMT = French-language medical multi-terminology indexer

† Abbreviation, spelling, synonym 
Table 3 Evaluation of reasons for coding false positives concepts by type of variable on the test set

\begin{tabular}{|c|c|c|c|c|c|c|c|c|c|c|c|c|c|c|c|c|c|c|}
\hline & \multicolumn{3}{|c|}{ Chief complaint } & \multicolumn{3}{|c|}{ Observation } & \multicolumn{3}{|c|}{ Specialists' notes } & \multicolumn{3}{|c|}{ Procedures } & \multicolumn{3}{|c|}{ Prescriptions } & \multicolumn{3}{|c|}{ Total } \\
\hline & $\mathrm{N}^{*}$ & $\%$ & $95 \% \mathrm{Cl}$ & $\mathrm{N}^{*}$ & $\%$ & $95 \% \mathrm{Cl}$ & $\mathrm{N}^{*}$ & $\%$ & $95 \% \mathrm{Cl}$ & $\mathrm{N}^{*}$ & $\%$ & $95 \% \mathrm{Cl}$ & $\mathrm{N}^{*}$ & $\%$ & $95 \% \mathrm{Cl}$ & $\mathrm{N}^{*}$ & $\%$ & $95 \% \mathrm{Cl}$ \\
\hline Temporality of event not recognized (antecedents) & 0 & - & - & 157 & 35.8 & $31.3-40.5$ & 0 & - & - & 0 & - & - & 0 & - & - & 157 & 35.4 & 31.0-40.1 \\
\hline False disambiguation of concept & 2 & 66.7 & $9.4-99.2$ & 253 & 57.8 & $53.0-62.4$ & 1 & 100 & - & 1 & 100 & - & 0 & - & - & 257 & 58.0 & $53.3-62.7$ \\
\hline Negation not detected & 0 & 0 & - & 19 & 4.3 & $2.6-6.7$ & 0 & - & - & 0 & - & - & 0 & - & - & 19 & 4.3 & $2.6-6.6$ \\
\hline False disambiguation of abbreviation or acronym & 1 & 33.3 & $0.8-90.6$ & 6 & 1.4 & $0.5-3.0$ & 0 & - & - & 0 & - & - & 0 & - & - & 7 & 1.6 & $0.6-3.2$ \\
\hline Other UrgIndex error & 0 & - & - & 3 & 0.7 & $0.1-2.0$ & 0 & - & - & 0 & - & - & 0 & - & - & 3 & 0.7 & $0.1-2.0$ \\
\hline Total number of false positive concepts & 3 & 100 & - & 438 & 100 & - & 1 & 100 & - & 1 & 100 & - & 0 & & - & 443 & 100 & - \\
\hline
\end{tabular}

Urglndex- Processing of emergency services medical narrative records (DMU) of the University of Lyon's Hôpital de la Croix-Rousse, Lyon, France

${ }^{*} \mathrm{~N}=$ Number of evaluated concepts 
cumulative sum algorithm. This system was able to prospectively reveal the onset of the flu epidemic earlier than the Sentinel Influenza Network, a routine surveillance system [7].

Studies have already been undertaken on the use of natural language processing in the syndromic surveillance system. Among them, a trial called Real-time Outbreak and Disease Surveillance (RODS) was conducted in 200 emergency structures in Pennsylvania, Utah, Ohio and New Jersey [6]. A free text extractor named CoCo (Complaint Coder) analyzed the chief complaints and automatically classified them according to naive Bayesian classification algorithms based on 1 of the following 8 syndromes: respiratory, botulism, gastrointestinal, neurological, cutaneous, constitutional, haemorrhagic and other. A detection algorithm then analyzed cluster research data. This system allowed the prospective detection of exposure to carbon monoxide [22]. A retrospective study at the University of Pittsburgh Medical Center ED evaluated the performance of the CoCo free text extractor [23]. The authors measured the extractor's ability to classify 527,228 patients admitted between 1990 and 2003 based on 1 of 7 syndromes: respiratory, botulism, gastrointestinal, neurological, cutaneous, constitutional and haemorrhagic. Each primary discharge diagnosis, already coded in ICD-9, was also retrieved and served as the "gold standard" to evaluate the extractor's performance. According to the results, the tool's sensitivity ranged from $30 \%$ for botulism syndrome to $75 \%$ for haemorrhagic syndrome. Its specificity was between $93 \%$ and $99 \%$.

Another example of a syndromic surveillance system with textual processing of chief complaints is that of the New York City Department of Health and Mental Hygiene, which uses another type of classification tool for chief complaints: their classification algorithm is based on a search of keywords [4]. The studied syndromes are common colds, infectious conditions or death upon arrival, respiratory syndromes, diarrhoea, fever, rash, asthma and vomiting. Abnormal events are detected by temporal and spatial clustering methods.

South et al. reported the value of employing multiple textual sources from computerized ED records, and not the sole chief complaint, to improve the ability to identify flu-like syndromes [24]. Indeed, the sensitivity of a free textual extractor in identifying patients admitted to EDs with a flu-like syndrome was $27 \%$ when the free textual extractor was applied to data on the chief complaint, $51 \%$ when applied to ED observation data, and $4 \%$ when applied to the triage nurse's observation data. By combining these various natural language data, sensitivity was increased to $75 \%$.

Authors have begun to focus on syndromic surveillance for nosocomial infection monitoring and alerts $[25,26]$. These trials exploit the computerized medical records of hospitalized patients to detect the beginning of intra-hospital outbreaks (e.g., gastroenteritis due to Norovirus). However, we have not found any articles on the use of syndromic surveillance data from EDs to implement an intra-hospital alert system for patients who could be an epidemic risk. The information provided by InVS surveillance systems, both nonspecific and specific to certain syndromes (the Sentinel Network for influenza and acute gastroenteritis, etc.) [27], is intended for regional and national surveillance. The information circuit for these systems is, therefore, not designed for intra-hospital purposes. The objective of syndromic surveillance within a hospital, as in a community, is to implement an appropriate alert for preventive measures that should be taken in a very reactive way in the facility during patient admission.

UrgIndex will be integrated into a clinical decision support system aimed at identifying cases of communityacquired infections with the aid of varied filtering of symptoms and procedures, but by customizing the filters, this application could also serve other types of clinical decision support systems: to assist in triage by directly processing the chief complaint for consultation; to help in diagnosis and management decisions; to participate in surveillance based on EDs and mortality (Surveillance Sanitaire des urgences et des décès, SurSaUD) in the InVS surveillance system by sending coded data (e.g., during summer heat wave periods, the InVS assesses the impact of heat waves on the population by analyzing the chief complaints for hyperthermia, dehydration, hyponatraemia and discomfort) [28]; to research case clusters during bioterrorism and to identify patients for rapid inclusion in study protocols.

\section{Conclusions}

UrgIndex, based on simple semantic analysis, automatically and effectively processes natural language data from ED records. An automated clinical decision support system, adopting such an application and integrated into hospital information systems, is an asset in preventing the risk of hospital infections, specifically by allowing the early identification of patients who pose an epidemic risk.

\section{Acknowledgements}

The authors thank Ovid Da Silva of IRTC Inc. and Joanne Zinkewich for their valuable translation and editing of this manuscript.

\section{Author details}

${ }^{1}$ Hospices Civils de Lyon, Hôpital de la Croix-Rousse, Unité d'hygiène et d'épidémiologie, F-69317 Lyon, France. ${ }^{2}$ Université de Lyon, F-69000 Lyon; Université Lyon 1; CNRS, UMR5558, Laboratoire de Biométrie et Biologie Evolutive, F-69622 Villeurbanne, France. ${ }^{3}$ Hospices Civils de Lyon, Direction Système d'Information et Informatique, F-69500 Bron, France. ${ }^{4}$ Hospices Civils de Lyon, Hôpital de la Croix-Rousse, Service des urgences, F-69317 Lyon, France. ${ }^{5}$ CISMeF, LITIS EA 4108 - Université de Rouen, F-76031 Rouen cedex, France. 


\section{Authors' contributions}

SG built the correspondence tables for non-standard terms and filters, and undertook the analysis. The application itself was designed and constructed by OY and QG. SD performed ECMT algorithms. ALM and VS participated in data collection with the DMU's data warehouse. SG, VP and MHM evaluated and determined which pertinent infectious disease to detect. SG drafted the manuscript and MHM revised it. All authors have read and approved the final manuscript version.

\section{Competing interests}

The authors declare that they have no competing interests.

Received: 7 February 2011 Accepted: 28 July 2011

Published: 28 July 2011

\section{References}

1. Comité technique des infections nosocomiales et des infections liées aux soins (CTINILS): Définition des infections associées aux soins Ministère de la santé, de la jeunesse et des sports DGS/DHOS; 2007, 11.

2. Henning KJ: What is syndromic surveillance? MMWR Morb Mortal Wkly Rep 2004, 53(Suppl):5-11.

3. Knirsch CA, Jain NL, Pablos-Mendez A, Friedman C, Hripcsak G: Respiratory isolation of tuberculosis patients using clinical guidelines and an automated clinical decision support system. Infect Control Hosp Epidemiol 1998, 19:94-100.

4. Heffernan R, Mostashari F, Das D, Karpati A, Kulldorff M, Weiss D: Syndromic surveillance in public health practice, New York City. Emerg Infect Dis 2004, 10:858-64.

5. Paladini M: Daily Emergency Department Surveillance System - Bergen County, New Jersey. MMWR Morb Mortal Wkly Rep 2004, 53(Suppl):47-9.

6. Tsui FC, Espino JU, Dato VM, Gesteland PH, Hutman J, Wagner MM: Technical description of RODS: a real-time public health surveillance system. J Am Med Inform Assoc 2003, 10:399-408.

7. Yuan CM, Love S, Wilson M: Syndromic surveillance at hospital emergency departments-southeastern Virginia. MMWR Morb Mortal Wkly Rep 2004, 53(Suppl):56-8.

8. Josseran L, Nicolau J, Caillere N, Astagneau P, Brucker G: Syndromic surveillance based on emergency department activity and crude mortality: two examples. Euro Surveill 2006, 11:225-9.

9. Balter S, Weiss D, Hanson H, Reddy V, Das D, Heffernan R: Three years of emergency department gastrointestinal syndromic surveillance in New York City: what have we found? MMWR Morb Mortal Wkly Rep 2005, 54(Suppl): $175-80$.

10. Mikosz CA, Silva J, Black S, Gibbs G, Cardenas I: Comparison of two major emergency department-based free-text chief-complaint coding systems. MMWR Morb Mortal Wkly Rep 2004, 53(Suppl):101-5.

11. Reis BY, Mandl KD: Integrating syndromic surveillance data across multiple locations: effects on outbreak detection performance. AMIA Annu Symp Proc 2003, 549-53.

12. Begier EM, Sockwell D, Branch LM, Davies-Cole JO, Jones LH, Edwards L, Casani JA, Blythe D: The National Capitol Region's Emergency Department syndromic surveillance system: do chief complaint and discharge diagnosis yield different results? Emerg Infect Dis 2003, 9:393-6.

13. Lazarus R, Kleinman KP, Dashevsky I, DeMaria A, Platt R: Using automated medical records for rapid identification of illness syndromes (syndromic surveillance): the example of lower respiratory infection. BMC Public Health 2001, 1:9.

14. Hutwagner L, Thompson W, Seeman GM, Treadwell T: The bioterrorism preparedness and response Early Aberration Reporting System (EARS). J Urban Health 2003, 80:189-96.

15. Ministère de la santé et des solidarités: Décret no 2006-576 du 22 mai 2006 relatif à la médecine d'urgence et modifiant le code de la santé publique (dispositions réglementaires). Journal Officiel de la République Française 2006

16. Société Française de Médecine d'Urgence, Ministère de la Santé et des Solidarités, et Institut de Veille Sanitaire: Résumé de Passage aux Urgences (RPU). 2006

17. Société Française de Médecine d'Urgence: Thésaurus des motifs de recours en structure d'urgence. [http://www.sfmu.org/documents/File/ referentielsSFMU/Motifs_de_recours_SFMU_2010.pdf].
18. Sakji S, Thirion B, Dahamna B, Darmoni SJ: [Searching French institutional health information sources: Catalogue and index of French-language medical sites (CISMeF)]. La Presse Médicale 2009, 38:1443-1450.

19. Soualmia L, Dahamna B, Thirion B, Darmoni S: Strategies for health information retrieval. Stud Health Technol Inform 2006, 124:595-600.

20. Pereira S, Névéol A, Kerdelhué G, Serrot E, Joubert M, Darmoni SJ: Using multi-terminology indexing for the assignment of MeSH descriptors to health resources in a French online catalogue. AMIA Annu Symp Proc 2008, 586-590.

21. Sakji S, Gicquel Q, Pereira S, Kergourlay I, Proux D, Darmoni S, Metzger M-H: Evaluation of a French medical multi-terminology indexer for the manual annotation of natural language medical reports of healthcareassociated infections. Stud Health Technol Inform 2010, 160:252-256.

22. Wagner MM, Espino J, Tsui FC, Gesteland P, Chapman W, Ivanov O, Moore A, Wong W, Dowling J, Hutman J: Syndrome and outbreak detection using chief-complaint data-experience of the Real-Time Outbreak and Disease Surveillance project. MMWR Morb Mortal Wkly Rep 2004, 53(Suppl):28-31.

23. Chapman WW, Dowling JN, Wagner MM: Classification of emergency department chief complaints into 7 syndromes: a retrospective analysis of 527,228 patients. Ann Emerg Med 2005, 46:445-55.

24. South BR, Chapman WW, Delisle S, Shen S, Kalp E, Perl T, Samore MH, Gundlapalli AV: Optimizing A Syndromic Surveillance Text Classifier for Influenza-like Illness: Does Document Source Matter? AMIA Annu Symp Proc 2008, 2008:692-696.

25. Kawana A, Teruya K, Kirikae T, Sekiguchi J, Kato Y, Kuroda E, Horii K, Saito S, Ohara H, Kuratsuji T, Kimura S, Kudo K: « Syndromic surveillance within a hospital » for the early detection of a nosocomial outbreak of acute respiratory infection. Jpn J Infect Dis 2006, 59:377-9.

26. Kikuchi K, Ohkusa Y, Sugawara T, Taniguchi K, Okabe N: [Preliminary trial of syndromic surveillance to early detection of nosocomial infection outbreak]. Kansenshogaku Zasshi 2007, 81:162-72.

27. Réseau Sentinelle. [http://websenti.b3e.jussieu.fr/sentiweb/ ].

28. Josseran L, Fouillet $A$, Caillère N, Brun-Ney D, llef D, Brucker G, Medeiros $H_{1}$ Astagneau P: Assessment of a Syndromic Surveillance System Based on Morbidity Data: Results from the Oscour ${ }^{\circledR}$ Network during a Heat Wave. PLoS One 2010, 5:e11984.

\section{Pre-publication history}

The pre-publication history for this paper can be accessed here: http://www.biomedcentral.com/1472-6947/11/50/prepub

\section{doi:10.1186/1472-6947-11-50}

Cite this article as: Gerbier et al.: Evaluation of natural language processing from emergency department computerized medical records for intra-hospital syndromic surveillance. BMC Medical Informatics and Decision Making 2011 11:50.

\section{Submit your next manuscript to BioMed Central and take full advantage of:}

- Convenient online submission

- Thorough peer review

- No space constraints or color figure charges

- Immediate publication on acceptance

- Inclusion in PubMed, CAS, Scopus and Google Scholar

- Research which is freely available for redistribution 